\title{
SINCE/BECAUSE ALTERNATION: INSIGHTS FROM CLAUSE STRUCTURES IN NIGERIAN ENGLISH
}

\author{
Mayowa Akinlotan \\ Catholic University of Eichstaett-Ingolstadt, Germany
}

\begin{abstract}
The choice between since and because allows language users to provide rationality which is part of the cognitive functions of language. Different conditions have been shown to explicate this alternation, with little attention paid to the clausal weight. The present paper shows how expression of rationality is alternated between choosing a since or because, since both have the semantic capacity to do so, in certain contexts. The study uses a simple measurement method to show the extent to which clausal weight relates to this alternation. Relying on corpus data from a well-known variety representing Nigerian English, the present study shows that the choice between since and because is related to a number of factors such as the type of text producing the usages. With 1074 usages showing such interchangeable usages extracted from academic and media text types in written Nigerian English, it is shown that, at least in the variety under examination, the choice of since over because as a rationality expresser is scarce, and that overall pattern can be predicted on the basis of certain contexts including clausal weight and ordering pattern. The scarcity of since as a rationality expresser is perhaps a reflection of interference from the local languages, which do not have semantic equivalents.
\end{abstract}

Key words: since/because alternation, syntactic alternation, rationality clauses, clause structure, structural variability

\section{Article history:}

Received: 4 August 2021

Reviewed: 30 September 2021

Accepted: 30 November 2021

Published: 30 December 2021

Copyright (C) 2021 Mayowa Akinlotan

This open access article is published and distributed under a CC BY-NC 4.0 International License which permits non-commercial use, distribution, and reproduction in any medium, provided the original author and source are credited. Permissions beyond the scope of this license may be available at mayowa.akinlotan@ku.de. If you want to use the work commercially, you must first get the authors' permission.

Citation: Akinlotan, M. (2021). Since/Because Alternation: Insights from Clause Structures in Nigerian English. English Studies at NBU, 7(2), 167-186. https://doi.org/10.33919/esnbu.21.2.3

Mayowa Akinlotan, PhD, is a research fellow of the Alexander von Humboldt Foundation and conducts research at the Catholic University of Eichstatt-Ingolstadt, Germany. He is also currently a research scholar at the Linguistics Research Center of the University of Texas at Austin, USA. He has published extensively on syntactic structures of varieties of English, using basic and advanced corpus methods of linguistic analysis.

E-mail: mayowa.akinlotan@ku.de

https://orcid.org/0000-0001-5142-7168 
One of the infinite flexibilities of languages, including all its varieties, is its creative aptitude to provide language users with syntactic choices such that language users can say the same thing with different usages. Some of these syntactic choices are a clear cut syntactically driven alternation (for example, genitive alternation in John's book vs. Book of John, and prepositional placement in we rely on this information versus this information we rely on) while some are a clear cut semantically driven creative alternation, one of which is the expression of rationality with a choice of since or because. The alternation between since and because is not as clearly cut as genitive alternation or prepositional placement or particle placement. In (1), the alternation of since/because is syntactically compatible but not semantically so. This is because since expresses just the fact, which is a matter of time, whereas because appears to have expressed a matter of reason. Consider that (1) provides rationality, which cannot be expressed with because, as in 'Because I was eight I have been playing golf'.

(1) Since I was eight I have been playing golf.

(2) Sadly, the Police have failed to discharge the onus of disproving this suspicion, since none of the cases has been conclusively investigated, years and months after

(3) After the Farnborough unveiling, aviation experts promptly adjudged the 81 routes as unrealistic because many existing airlines with large fleets fly to Nigeria which is one of the most lucrative destinations for airlines.

Whereas in (2) and (3), because and since are interchangeable such that meaning and structure are not impeded. Unlike (1), both (2) and (3) cognitively express argumentation structure where since and because serve as signifier of premise from which conclusion is made. The argumentative structure infused in the meaning of the dependent and independent clauses means that the basic meaning of since and because in (2) and (3) is that of rationality. As can be deducted, the alternation of since/because usage thus becomes a matter of creativity, structure, meaning and cognition which deserves much more attention than it has received when compared to less complex alternations such as genitive, preposition, and particle placement. Also, it becomes more important to find out the structural patterning and scenarios characterising the since/because rationality alternation because same/similar variables explaining usage choice in these clear-cut alternations have been found behaving differently in new varieties of English from those of established varieties. 
Given that there is little or no serious empirical investigation into this alternation of since/because rationality from new varieties of English, the present study thus aims at providing the basic threshold of data and investigation, showing how speakers/users of new varieties alternate with use the of since and because in expressing rationality. A corpus material of 1.1. million length were queried, resulting into 1074 interchangeable usages that are quantitatively ad qualitatively analysed against eight variables representing (1) genre/text type (academic versus media writing), (2) length of clause I (3) length of clause II (4) structural ordering; main + dependent versus dependent + main order (5) expression of causality or conditionality (6) sentence length (7) dependent clause length, and (8) main clause length. A variationist/distributional analysis smoothly blended with perspectives from cognitive usage-based and usage grammar show that there is some systematicity involved, and clearly provide specific contexts when and why certain usages are more likely to occur. Among many findings academic text type is expected to be more characterised with the choice of because while media text type is expected to be characterised more with the choice of since.

\section{Since and because alternation and contexts of usages}

The questions of syntactic alternation, together with different interplays of variables and cognition on different usage choices continued to be addressed in the literature. The alternation between since and because clauses have been studied in the light of causal adverbial clauses, together with different other semantic categories of clauses that constrained the expression of causality, conditionality, and rationality (Altenberg 1984; Kanetani 2007, 2019; Diessel 2005, 2008; Couper-Kuhlen \& Kortmann, 2000). More specifically, different sorts of evidence of usage choices instantiating the relation between cognition, grammatical usage, and meaning making have been put forward. According to Goldberg (1995), usages can be syntactically distinct and semantically synonymous. Evidence from dative alternation, genitive alternation, participle placement, and many others have indeed shown the relation, working and variability of syntactically distinct but semantically synonymous usages.

If according to Kachru (2019), the distinctiveness and peculiarities of different varieties of English, especially those divided along the inner circle ones (i.e. American, 
British, Canadian) and outer circle ones (for example, Nigerian, Indian, Singaporean, Ghanaian) continued to be established, then one must expect different workings of the systems operating syntactic alternations in different varieties. More specifically, Akinlotan $(2017,2018)$ have shown how usage patterns in Nigerian variety of English differ from that of British and American varieties with which it shared formal properties. More recently, Akinlotan (2020) shows how contextual usages of cognitively driven BE-relativisation differ in Nigerian and Canadian varieties of English. One of such alternations, which is yet to receive serious attention in new varieties of English, is the usage choice between since and because expressing causality, conditionality, and reasonability. Kanetani $(2006,2019)$ argues that usage involving because can be distinguished into two types: those that express causality and those that express conditionality.

According to Sawada (2004), in constructing because usage, native speakers of established varieties of English such as British and American, prefer it is because usage. Sawada asserts that this choice is preferred because it strengthens the exclusivity of the reason being provided for the action being reported. According to Sawada, preferring such usage implies that the speaker intends to cancel out any other possibility of a reason for the action being reported. In the literature, it is generally agreed that usage choices between since and because, and expression of causation and rationality/conditionality is related to a number of variables representing syntactic, semantic, pragmatic cognitive and discourse perspectives. More specifically, Kanetani (2019), Diessel (2005, 2008), Hetterle (2015), Diesel \& Hetterle (2011), Dancygier 1998, Dancygier \& Sweetser (2000) have found that causal relations and reasoning processes, both of which are clearly possible by since and because usages, are related to communicative function and the relative position of the main clause.

More so, it is expected that such alternation between since and because in expressing causation and rationality/conditionality will be related to the processing theory of constituency and order by Hawkins (1994, 2004), together with the principle of end-weight (Wasow, 2002), both of which argue that syntactic choices, such as the position of a syntactic unit, greatly influence how reader/hearer process them. Dancygier \& Sweetser (2000) examined the competition between since and between, showing how a number of variables relating to cognitive and discourse perspective 
influence use, form, meaning and interpretation. Similarly, Diessel \& Hetterte (2011) conducted a cross-linguistic investigation into the structure, meaning and use of causal clauses. They showed that communicative function, positioning of the dependent clause, and the meaning of the clause influence usage choices. For instance, it is found that clausal clauses predominantly followed main clauses. Also, Diessel $(2001,2005)$ found that meaning of the adverbial clause affects the ordering of main and dependent clauses.

\section{Corpus extraction and preliminary description}

Data were drawn from the Nigerian component of the International Corpus of English (ICE) and from additional text types matching those in ICE. For instance, academic and media texts from ICE-Nigeria were supplemented by collecting more academic and media texts that followed the guidelines used in ICE. Journal articles written were collected from journals, while media texts such as editorials and news writings were collected from local Nigerian newspapers such as the Vanguard, the Guardian, and the Punch. The additional text types collected reached a million wordlength, which is about the size of each regional component in ICE. Then usages consisting of all uses of since and because were first extracted using AntCon. Initial extraction consists of all of such usages that border around causality, rationality or conditionality is expressed. The data were then manually read again to retain only instances where since and because are used to express rationality. Consider (4) and (5).

(4) Similarly, Mandela never saw borders in Africa since the whole continent won freedom for him and his country.

(5) Since an idol is not a mere mortal like the rest of us, they also brought along the necessary items for appeasing dedidu, who was at his best dispensing political favours

For examples, 4-7 express rationality and conditional while 8-9 do not. A test of substitution is applied. I substituted every instance of since with because, and vice versa, checking for syntactic and semantic compatibility such as ensuring that they allow smooth reading expressing rationality, conditionality or causality. In (4-7) since and because can be well interchanged. Although since has two basic inherent meanings involving reason and time (i.e. since $=+$ reason $(+)+$ time), it is often used in the sense of because, which can have some sense of time infused into its reason (i.e. because $=+$ 
reason* (x since): which means that the expression of time by because is often implicit unlike that of since). In (4) since clearly expresses reason and time, where (5) expresses only reason. However, because can perfectly fit into both usages. Same permutation is possible in (6) and (7), in which since can perfectly fit into because without interference into the meaning processing. In (7), because of can be interchanged with since there is a.

(6) The publicity will be done at the community level but national medium are included because it is important that all Nigerians are aware of the amnesty policy, appreciate the message of peace, and collectively participate in the peace process.

(7) Amnesty should be pursued pari-passu with effective anti-bunkering measures because of the close correlation between illegal oil bunkering and armed conflict in the Niger Delta region

This test of substitution thus implies that only a careful reading can allow extraction of such since/because alternation, and that a careful attention is paid to the expressibility of reason, such that a basic question of why can be answered by a use of since or because. Hence only instances such as (4-7) were used for the analyses contained here. In the next stage involving annotation, a total of 1074 instances which qualify as since/because alternation were then annotated on the basis of eight variables representing (1) weight/length of the since/because clause, which is also the dependent clause (2) weight/length of the main clause (3) genre/text type - academic versus media writing (4) the weight/length of the entire sentence consisting of the dependent and main clauses, (5) length of the clause in the first position, which can be dependent or main, (6) length of the clause in the second position, (7) type of meaning being expressed which is classified as being causality or not causality, and (8) ordering of the clause structure (i.e. main + dependent or dependent + main ordering).

For instance, in (8), the clause in the first position is The resignation was forced, which is a main clause while the clause in the second position is because the people showed interest in what happened at their parliament, a dependent clause. The length of these clauses in terms of position they occur is also accounted for. For example, the length of the clause in first position or clause I (i.e. the resignation was forced) is threeword-length, while that of clause II (because the people showed interest in what 
happened at their parliament) is seven-word-length. Akinlotan $(2017,2018)$ has shown that complexity, which can be measured in different dimensions such as weight, length, or structural node, is significantly related to usage choices in syntactic alternation in new varieties of English, and especially in Nigerian variety of English. These findings also support the findings by Hawkins (1994), Wasow (2002), and Rosenbach (2005) that weight/complexity/length is very much related to syntactic choices and usages. More so, Dessel $(2001,2005,2008)$ has shown that ordering and differential positioning of dependent and main clauses within the sentence structure is significantly related to the type of meaning being expressed as well as the structural complexity in the dependent/main clause and the complexity in the entire sentence structure.

(8) The resignation was forced because the people showed interest in what happened at their parliament.

The word-length of every clause is accounted for, leaving out preposition, conjunctions, articles/determiners, and non content words. For instance, the weight of the dependent clause because the people showed interest in what happened at their parliament in (8) is 7 while the length of the main clause the resignation was forced is 3 , and the total length of the sentence thus sums up as 10 . Also, each usage is classified as expressing causality or non-causality. I followed the binary distinction used by Kanetani $(2007,2019)$ and Dancygier \& Sweetser (2000) where clear distinctions were made between since/because usages that express causality and those that do not. Such usage that do not express causality might express other conditions such as contrast, conditionality, time, and rationality which does not necessarily bring about a causeeffect relationship.

Following this line of thought, all the usages are then classified into causal and non-causal usages. Another important variable accounted for is that of genre/text type. Akinlotan $(2017,2018)$ has repeatedly shown the importance of text type/genre in understanding structural patterning and usage choices in new varieties of English, especially in Nigerian variety of English. Following this finding, it is also expected that the variable representing genre/text type will explain the variability and usage choices involved in the use of since/because for the expression of reason. Table 1 shows the overview distribution of sample used for the analyses. As can be seen in Table 1, since and because are evenly distributed across the academic and media text type. 
Table 1

An overview distribution of since/because usages

\begin{tabular}{lllllll} 
& \multicolumn{2}{c}{ since } & \multicolumn{2}{c}{ because } & \multicolumn{2}{l}{ Total } \\
& $\boldsymbol{N}$ & $\%$ & $\boldsymbol{N}$ & $\boldsymbol{\%}$ & $\boldsymbol{N}$ & $\%$ \\
\hline Academic text type & 267 & 49 & 274 & 51 & 541 & 100 \\
\hline Media text type & 262 & 49 & 271 & 51 & 533 & 100 \\
\hline Total & 529 & 49 & 545 & 51 & 1074 & 100
\end{tabular}

As it will be shown in the next section, the distributions were then analysed in terms of the eight variables representing length of the since/because clause, length of the main clause, length of clause in the first and second position, length of the entire sentence, expression of causality, and genre. Results of the distributional analyses, together with qualitative interrogation, are presented in the following section. A test statistic chi square test of independence is used to test out the relationship between the usage choice and the variables under studied.

\section{A simple distributional analysis}

In this section the results of the annotation and preliminary analyses are presented and further discussed in the light of the expectations in the literature and contexts of emerging patterns. As can be seen in Table 2 the distribution of the alternation in terms of how each variable independently influence usage choice is provided. Such explanation is important because it helps to understand how, and the extent to which, variability is related to these different determinants. The distribution of length between long, medium and short is conceptually operationalised by dividing the longest length by 3 . For instance, the longest main/dependent clause length found in the data is that of 29 word-length, which when divided by 3 gives a score of 9.6.

Hence, the short length is between 0 to 10 , medium length between 11 to 20, and long length is 21 word-length and above. In the same vein, the longest length of the sentence structure (i.e. main + dependent clause sum up) is that of a 38-word length sentence, which when divided by 3 , gives 12.6 word-length, such that short sentence has 0-13 word-length, medium sentence 14-26, and 27 word-length sentence structure refers to the long sentence types. Using independent procedure in such classification ensures objectivity. 
Table 2

A bivariate table showing distribution between since and because

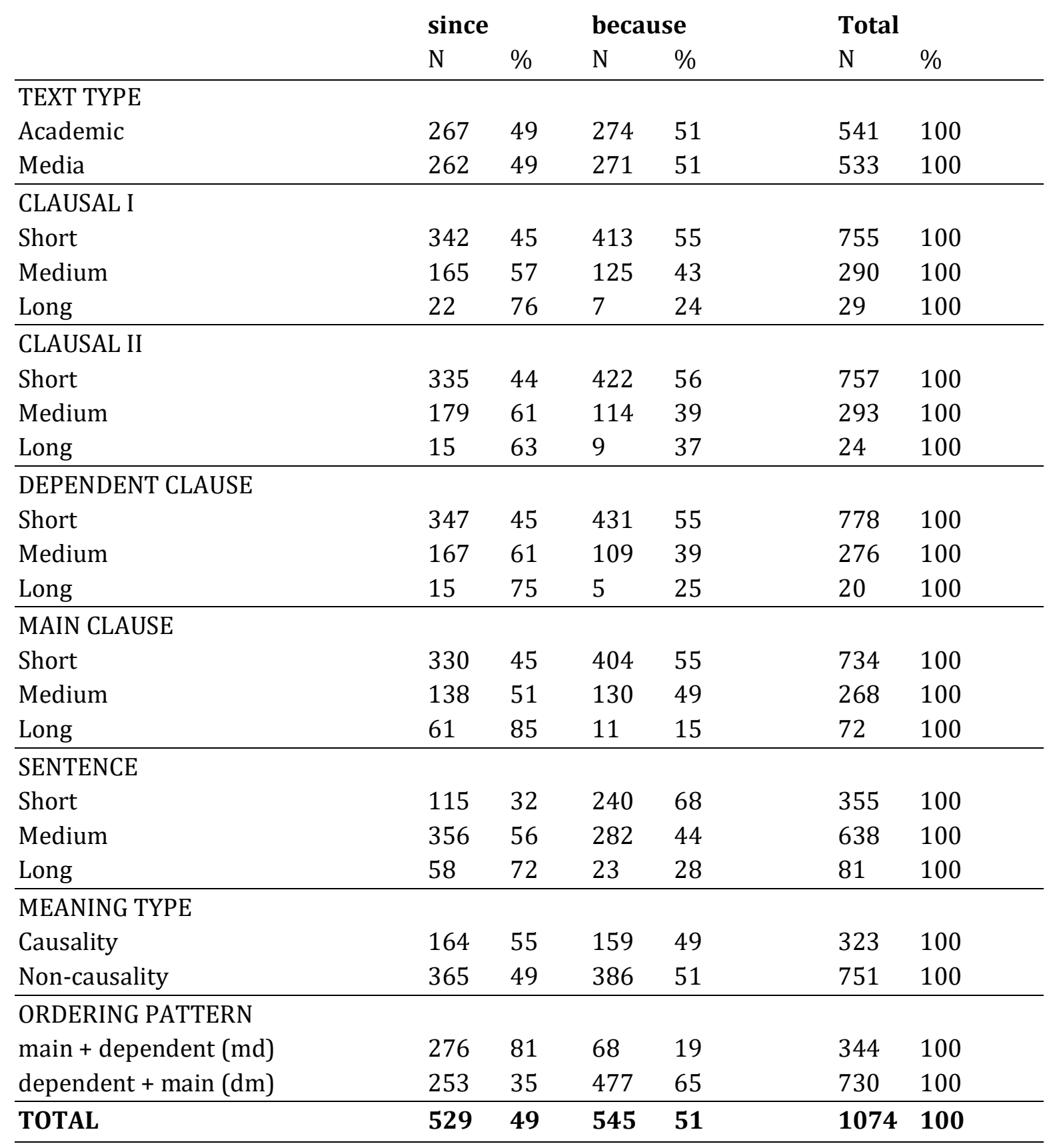

In order to test out the independent behaviour of each variable in terms of influencing a usage choice between since and because, a series of chi square test of independence was carried for each variable. Chi square test of independence allows to see whether choosing a since or because usage is related to each of these variables. A chi square test shows that text type/genre is not significantly related to since/because alternation. The relation between choosing since/because and text type that produces the usage was insignificant, $\mathrm{X} 2(1, \mathrm{~N}=1074)=0.004, \mathrm{p}=0.95$. As can be seen in Table 2 , 
the choices between since and because are equally distributed, which shows that the distinction between academic and media text types is not sufficient to provide explanation for the variability in the two usages.

This result might not be so surprising given that Akinlotan (2017) has shown media and academic writings to be close representative of standard Nigerian English, in which certain syntactic characteristics are demonstrated. In other words, the distinction between academic or media text type in our data is not able to explain the variability or usage choice pattern. This result follows Akinlotan \& Housen (2017) where genre/text type has been found out weighted by syntactic determinant in explaining structural variability in Nigerian variety of English. The strong influence of genre/text type as a determinant of usage choice has been emphasised in the literature, though this result further shows that such strength is dependent on the linguistic variable being studied.

Another variable not statistically significant in influencing usage choice is the expression of meaning contained in the alternation. The relation between expression of meaning and usage choice is insignificant, $\mathrm{X} 2(2, \mathrm{~N}=1074)=0.426, \mathrm{p}=0.514$. As can be seen in Table 2, expression of causality and non-causality does not show a pattern with which choices between the two usages can be predicted. Of course, such finding might reflect the need, in further studies, to tease apart the binary classification such that usages expressing non-causality can be further classified. Nevertheless, since the literature agrees that expression of causality is inherent semantic characteristic of such alternation, one would have expected some tendencies pointing towards such expectation. In other words, the finding might as well be a reflection of different usages of since/because alternation in our variety, given transfer of interference from similar usages that exist in local co-existing languages of Yoruba, Igbo and Hausa.

Kanetani (2007, 2019) and Dancygier \& Sweetser (2000) have shown that since/because alternation expresses a variety of meaning including expression of causality, conditionality, and rationality, although they have not sought out the relation between the alternation and expression of meaning. Kanetani (2019) and Goldberg (1995) have noted that syntactically different usages do indeed express synonymous meaning, which according to Kanetani (2019) can help us predict the two usages. For 
instance, (9-12) express alternation of meaning. As can be interpreted (10) and (11) which are interchangeable uses of since and because express the same intent of meaning, which is the expression of causality.

(9) Since most of the people that adopt this mode of transport are traders, they pack more goods than the old and rickety boats can carry.

(10) Police authorities should be conscious of this: investigation should not take too much time since the suspect has admitted that he is, indeed, the assailant in the video footage and the truth that is self-evident therein

(11) There is no doubt that Abiola's death left a void that nobody has been able to fill - not because there is lack of trying,

(12) If Nigeria can now boldly stake a claim to being a democracy, it is because some people paid the price.

In (10) the admittance of guilt by the suspect meant investigation should lead to prosecution quickly, and in (11) the death of Abiola led to death of sports sponsorship. Whereas (9) and (12) do not express causality but meaning-intent focusing on rationality and conditionality. Although distinctions relating to meaning and pragmatics can be shown, the test statistic shows relationship between distribution and contexts under examination.

\section{Clausal type and since/because alternation}

As can be seen in Table 2, all the variables representing length of clause I and II, dependent and main clauses, and sentence length provide insights into the operations involved in the two usage choices. Also, chi square test of independence further affirm that these variables are statistically significant, and can indeed explain how the choices between since and because usages work. The relation between since/because alternation and these variables are positive; length of Clause I $(X 2(2, N=1074)=$ 19.719, p=0.000); length of clause II (X2 (2, N = 1074) = 25.686, p=0.000); dependent clause length $(X 2(2, N=1074)=26.025, p=0.000)$; main clause length $(X 2(2, N=1074)$ $=42.193, \mathrm{p}=0)$; sentence length $(\mathrm{X} 2(2, \mathrm{~N}=1074)=67.497, \mathrm{p}=0)$; and ordering $(\mathrm{X} 2(2$, $\mathrm{N}=1074$ ) $=194.306, \mathrm{p}=0$ ). As Table 2 shows, since-usage is preferred to because-usage when Clause I is more likely to be longer, and because-usage preferred to since-usage when Clause I is more likely to be short. 
Given that since/because Clause I is providing explanation for action in Clause II, then it suggests that writers in our data chose since-usage when there are more information/explanation to be provided on the action in Clause II. In other words, when less information/explanation is being provided by the writer then because-usage is preferred. This finding relates to Hawkins' (1994) information structure, which can also allow to propose that the explicit inherent duality of meaning of time and reason in since means that bilinguals, who are under serious interference from competing equivalence in local languages, are likely to choose since-usage in order to explicitly express time and reason, usages that might be different from usages in established varieties. More so, the distribution in Clause II further affirm this pattern. In other words, irrespective of the position of because-usage, it is more likely to be structured shorter than since-usage. In (13) and (14), because-clause is placed in second and first positions, and both are shorter when compared to (15) and (16).

(13) In the US, it has become an epidemic because of the proliferation of guns in that society.

(14) Because lawmakers abuse their powers of appropriation, they lose the moral nerves to check the failings of the executive branch.

(15) Since there is high demand for USD which makes Naira to chase USD international trade, CBN should make USD to pursue Naira

(16) Going by the worsening electricity supply situation in the country, whatever measures could be applied to bring about change by way of improved supply is desirable especially since Nigerians had expected to see improved performance under the DISCOs regime so far to no avail.

In (15), there is a relative clause which makes Naira to chase USD international trade which is frequently not combined with because-clause in our data. The relative clause shows the tendency by the writer to respond to the explicit demand of since to provide more information and explanation that fit into the time and reason property of the since-usage. This cognitive tendency is perhaps reinforced by the usage of sinceclause in local Nigerian languages where reason-only alternation between since and because is rare. In another dimension I further investigated the relationship between the 
weight of the since/because bearing clause (i.e. the dependent clause) and the weight of the other clause (i.e. the independent clause). Relatedly, Table 2 shows that the overall length of the sentence can as well provide some explanations into the usage choices; because-sentence is more likely to be simpler/shorter than since-sentence. This pattern is a resultant effect already shown in the relation between clause positioning and length, and the tendency for since to express time and reason rather than reason only in because-usage.

As can be seen in Table 2, the tendencies for because-usage to be simpler than since-usage are again demonstrated in which since-dependent-clause (for example, 'since he could not pay the loan', in since he could not pay the loan, his company was taken over) and since-independent-clause (for example, his company was taken over) are more complex than both because-dependent-clause and because-independent clause. As can be seen, because-dependent clause (for example, 'because he overslept' in because he overslept, Shade missed the test) and because-independent clause (i.e. Shade missed the test) are repeatedly shorter (55\%) than since-dependent and independent clauses which are repeatedly medium and long lengths $(61 \%$ and $75 \%$, and $51 \%$ and $85 \%$ respectively.

Meanwhile the patterning becomes clearer when we examine the distributional pattern in ordering, a variable which also shows significant relation between since/because usage choice and the positioning/movement of dependent and independent clause. Table 2 shows that since-usage is more likely to have an MD structure, while because-usage is more likely to have DM structure. In other words, independent clause is more likely to be placed before since-dependent clause, whereas because-dependent clause is more likely to be placed before the independent clause. Given that both since-dependent and main-independent clauses are usually longer than both because-dependent and because-independent clauses, the movement of these clauses then appears a more systemic matter for rationality expression using because.

A cognitive explanation of a natural logic order in which premise should precede conclusion appears to underlie the DM structure in because-usage. A linear natural order in argumentation means that conclusion follows premise, and that if the premise is true, then the conclusion becomes explicit, inferable, and thus requires less scrutiny. Also, because is explicitly a tip-off to argument structure, just as since is explicitly a tipoff for time-in-reason. 

Because the people hate the policies of the President, they voted him out Since the people hate the policies of the President, they voted him out They voted the President out, since the people hate his policies They voted the President out, because the people hate his policies

Premise is placed before the conclusion in (17) and (18) while the conclusion is placed before the premise in (19) and (20). The argument, which is the people hate the policies, is a more of a matter reason than a matter of time in reason because how long the people have hated the policies of the President is less of importance, which implies that (17), which is a because-usage, is a better fit than (18) for such purpose. Furthermore, placing the premise before the conclusion provides a platform for cognitive interaction between the writer/speaker and the reader/hearer. In (17) and (18) the reader/hearer is able to predict a positive or negative conclusion on the basis of the premise stated. Such interaction is possible irrespective of whether since or because is used, although because-cognitive interaction will only seek out reason in conclusion while since-cognitive interaction will only seek out time and reason in conclusion.

\section{Text type and alternation}

According to the syntactic patterns found in Table 2, there is no enough distinction between academic and media writing to show that a certain usage is more preferred in a certain text over the other. Although the influence of genre/text type is not statistically significant as it were in the univariate analyses presented in Table 2, a different dimension involving bivariate analysis, together with a conceptualisation of genre/text type as a usage (Nikiforidou 2016; Hoffmann \& Bergs, 2018). Following this line of thought that genre/text type that is a usage, just as since/because usage, then the extent to which usage choices between since- and because- usage are related to academic-text type and media-text type usage can be become clearer. As can be seen in Table 3, which provides a more detailed and insightful relationship between genre/text type and the alternation, there is clearly a positive relationship between genre/text type and usage choices between since-usage and because-usage.

Table 3 
Alternation by genre, weight, causality, and ordering pattern

\begin{tabular}{l|ll|ll|ll}
\hline & \multicolumn{2}{|l|}{ since } & \multicolumn{2}{|l|}{ because } & \multicolumn{2}{l}{ total } \\
\hline academic & \multicolumn{2}{|l|}{} & & & & \\
\hline short & 49 & 33 & 101 & 67 & 150 & 100 \\
\hline medium & 194 & 55 & 156 & 45 & 350 & 100 \\
\hline long & 24 & 59 & 17 & 41 & 41 & 100 \\
\hline causality & 64 & 42 & 91 & 58 & 155 & 100 \\
\hline Non-causality & 203 & 54 & 183 & 46 & 379 & 100 \\
\hline Main +dependent & 139 & 82 & 31 & 18 & 170 & 100 \\
\hline Dependent+ main & 128 & 35 & 243 & 65 & 364 & 100 \\
\hline media & & & & & & \\
\hline short & 66 & 32 & 139 & 68 & 205 & 100 \\
\hline medium & 162 & 56 & 126 & 44 & 288 & 100 \\
\hline long & 34 & 85 & 6 & 15 & 40 & 100 \\
\hline causality & 100 & 60 & 68 & 40 & 168 & 100 \\
\hline Non-causality & 162 & 44 & 203 & 56 & 365 & 100 \\
\hline Main +dependent & 138 & 37 & 234 & 63 & 372 & 100 \\
\hline Dependent+ main & 124 & 77 & 37 & 23 & 161 & 100 \\
\hline & & & & & &
\end{tabular}

A chi square test statistic examining the relation between media/academic sentence length, media/academic meaning type, and media/academic ordering pattern and how these variables relate to choices between since/because usage, shows that there positive relationships. The relation between since/because alternation and these variables are positive; text type sentence length $(X 2(5, N=1074)=73.2, p=0.000)$; text type meaning type $(\mathrm{X} 2(3, \mathrm{~N}=1074)=16.2, \mathrm{p}=0.001)$; and text type structural ordering $(\mathrm{X} 2(3, \mathrm{~N}=1074)=176, \mathrm{p}=0.000)$. As can be seen in Table 3 , a complex/long-structured since-usage is more likely to be realised in a media text type than in an academic text type (85\% vs. 59\% respectively). On the other hand, a complex/long-structured because-usage is more likely to be realised in an academic text type than in a media text type (45\% vs. $15 \%$ respectively). Having earlier asserted that since-usage has a semantic tendency to be longer than because-usage, then it is reasonable to assume that the tendency for academic texts to prefer brevity correlates with preference for because-usage.

Furthermore, it can be seen that media writing is more likely to produce causality 
since-usage than academic writing ( $60 \%$ vs. $42 \%$ respectively), whereas academic writing is more likely to produce causality because-usage than media writing (58\% vs. $40 \%$ respectively). If we follow the line of thoughts in Hoffmann and Bergs (2018) and conceptualise genre/text type a separate usage, then the FORM-side of media writing ensures that there a mental MEANING-side mapping that answers the questions of WHAT:TIME; WHAT:WHY, and so on. The tendency for media/journalistic writing to ensure a FORM: MEANING mapping explains media writing's high preference for sinceusage expressing causality. In addition, as can be seen in Table 3 , because-usage expressing non-causality is more likely to be realised in media writing than in academic writing (56\% vs. $46 \%$ respectively). In other words, media writing automatically deselects causal because-usage, while academic writing prefers this usage.

Another clear distinction made between media and academic writing shows that certain text type prefers certain structural ordering. As can be seen in Table 3, academic text prefers to order since-usage as a main + dependent structure, whereas media writing prefers to order the same usage as dependent + main structure (82\% vs. 37\% respectively). On the other hand, media writing is more likely to order because-usage as a main + dependent structure, while academic writing is more likely to order the same usage as a dependent + main structure (63\% vs. $18 \%$ respectively). In other words, in academic writing when a since-usage is chosen, the dependent clause (i.e. the since-clause) is more likely to be placed in a second position, which is the opposite for choice of because.

(21) Focused interview of Muslim scholars and Imam's were conducted, to find out if the Muslims are generally pro-violence or pro-terrors, since concepts like extremists, fanatics, fundamentalists, separatists and Islamic militants are often used in describing the terrorists.

(22) Because the government is aware of this, it has to explain its case in enlightenment campaigns in euphemistic and optimistic terms, which other knowledgeable people outside the government may not share.

The above (21-22) usages are examples showing preferred ordering patterns from academic writing, in which because-clause in because usage is often placed in the first position while since-clause in since-usage is often placed in the second 
position. On the other hand, media writing does not seem to favour these ordering patterns. In other words, there will be some movement in the positions of clauses in (21) and (22), such that since-clause in since-usage is placed in the first position, and because-clause in because-usage placed in the second position. Of course such movement and positioning is underlined by number a syntactic, pragmatic, discourse, and cognitive factors. For example, Kanteni (2019) and Diessel (2005, 2008) have provided some explanation pointing towards the tendency by writer/speaker to place first that (reason or conclusion) which needs more focus. As can be seen in Table 3, this tendency to focalise that constituent of the narrative (i.e. reason + conclusion or conclusion + reason; cause + effect or effect + cause) is demonstrated in both academic and media writing, albeit the choice of usage to do so is significantly different between the text types.

\section{Concluding remarks and a semantic schema for since}

The present study has shown that alternation in the since/because rationality usage involves operations that are characterised with a combination of syntactic, semantic, pragmatic, discourse and cognitive factors all working simultaneously. The fact that only a small number of usage of since/because usage can actually interchange suggests that the kind of rationality alternation studied herein is less a matter of deliberate choice than it is for genitive alternation, preposition placement or particle placement. Unlike because which has only one meaning (i.e. the expression of reason), since has two meanings, the first being expression of reason and the other being the expression of time. In other words, unlike because which only equates + reason, since has capacity in itself to alternate between three usages.

(a) Since $=(+$ time $)+(+$ reason $)$

(b) Since $=(+$ time $)-$ reason

(c) Since $=(+$ reason $)-$ time, which $=$ because $\mathrm{x}(+$ reason $)$

Also, the present study has proposed a methodological test of substitution, which allows for the identification of all the variants of since- and because- usage expressing alternation in rationality. In other words, alternation in rationality usage is beyond structural composition, such that variants such as it is because, because of, because $x \mathrm{NP}$ are not syntactically but semantically equivalents of since, which is not morphologically 
variable enough to allow for such usage as it is since, and since of.

In addition to providing basic understanding on the nature and working of since/because alternation in new varieties of English, the study has clearly shown that the alternation is not conditioned by the semantic and pragmatic outputs of the usage. The study did not find a positive relation between meaning type (i.e. usages expressing causality or non-causality) and structural choice (i.e. since versus because). In other words, it is found that since- and because- usage freely alternate in expressing causeeffect actions and non-cause-effect actions which include conditionality. In (23), because lives are routinely violated by criminals masquerading as herdsmen simply proffer a premise explicating the conclusion placed in the main clause security is virtually nonexistent. Whereas in (24) there is a cause (i.e. the resignation of Professor Attahiru Jega as INEC Chairman) and effect (vacancy in the leadership of INEC).

(23) Security is virtually non-existent because lives are routinely violated by criminals masquerading as herdsmen.

(24) Since Prof. Attahiru Jega made good his promise not to continue with the job when his tenure expired in June, the position of the chairman of the electoral body has been vacant.

It is surprising that a semantically driven alternation is not found to be conditioned by the types of meaning being expressed. Unlike expression of causality/non-causality, the study found that since-because alternation is conditioned by (1) weight/length/structural complexity, a variable operationalised in different dimensions, structural ordering (i.e. main + dependent versus dependent + main order), and (3) genre/text type (i.e. structural patterns between academic and media writing). As can be seen in Table 3, and throughout the analyses which blend concepts from corpus-based and cognitive usage grammar, it is shown that text type/genre, in consideration of weight and structural order, might be the strongest variable that allows penetration into cognitive operation underlying the alternation of since/because rationality. Although the analyses presented here combined quantitative and qualitative measures, more data and analyses involving regression analyses might present a different scenario.

\section{References}


Akinlotan, M., \& Housen, A. (2017). Noun phrase complexity in Nigerian English: Syntactic function and length outweigh genre in predicting noun phrase complexity. English Today, 33(3), 31-38. https://doi.org/10.1017/S0266078416000626

Akinlotan, M. (2018). The Structural Simplification Hypothesis and the Premodifiers in Nigerian English. Anglica An International Journal of English Studies, 27(2), 58-88. https://doi.org/10.7311/0860-5734.27.2.04

Akinlotan, M. (2021). Calling a spade a shovel: a cognitive construction account of BErelativisation. Studia Neophilologica. https://doi.org/10.1080/00393274.2021.1980738

Altenberg, B. (2008). Clausal linking in spoken and written English. Studia Linguistica. A Journal of General Linguistics, 38(1), 20-69. https://doi.org/10.1111/i.14679582.1984.tb00734.x

Couper-Kuhlen, E., \& Kortmann, B. (2000). Cause - Condition - Concession - Contrast: Cognitive and Discourse Perspectives. De Gruyter. https://doi.org/10.1515/9783110219043

Dancygier, B. (1998). Conditionals and prediction. Cambridge University Press. https://doi.org/10.1017/CB09780511486463

Dancygier, B. \& Sweetser, E. (2000). Usages with if, since, and because: 585 Causality, Epistemic stance and clause order. In Couper-Kuhlen, E. \& Kortmann, B. (Eds.), Cause - Condition - Concession - Contrast: Cognitive and Discourse Perspectives (pp. 111-142). De Gruyter. https://doi.org/10.1515/9783110219043-006

Diessel, H. (2001). The Ordering Distribution of Main and Adverbial Clauses: A Typological Study. Language, 77(3), 433-455. https://doi.org/10.1353/lan.2001.0152

Diessel, H. (2005). Competing motivations for the ordering of main and adverbial 593 clauses. Linguistics, 43(3), 449-470. https://doi.org/10.1515/ling.2005.43.3.449

Diessel, H. (2008). Iconicity of sequence: A corpus-based analysis of the positioning of temporal adverbial clauses in English. Cognitive Linguistics, 19(3), 465-490. https://doi.org/10.1515/COGL.2008.018

Diessel, H., \& Tomasello, M. (2006). A New Look at the Acquisition of Relative Clauses. Language, 81(4), 882-906. https://doi.org/10.1353/lan.2005.0169

Diessel, H., \& Hetterle, K. (2011). Causal clauses: A cross-linguistic investigation of their structure, meaning and use. In Siemund, Peter (Ed.), Linguistic Universals and Language Variation, 21-52. De Gruyter.

Goldberg, A. (1995). Usages: A Usage Grammar Approach to Argument Structure. University of Chicago Press. 
Hawkins, J. (1994). A performance theory of order and constituency. Cambridge University Press. https://doi.org/10.1017/CB09780511554285

Hawkins, J. (2004). Efficiency and Complexity in Grammars. Oxford University Press. https://doi.org/10.1093/acprof:oso/9780199252695.001.0001

Hetterle, K. (2015). Adverbial Clauses in cross-linguistic perspective. De Gruyter. https://doi.org/10.1515/9783110409857

Hoffmann, T. \& Bergs, A. (2018). A Construction Grammar Approach to Genre. CogniTextes 18. https://doi.org/10.4000/cognitextes.1032

Kachru, B. (2019). 'World Englishes and Culture Wars.' In C. L. Nelson, Z. G. Proshina \& D. R. Davis (Eds.), The Handbook of World Englishes (pp. 447-471). Cambridge University Press. https://doi.org/10.1002/9781119147282.ch25

Kanetani, M. (2005). Relations among Usages with Because: With Special Reference to Metalinguistic Uses of Because, Tsukuba English Studies, (24), 31-50.

Kanetani, M. (2006). Usages of Causation and Reasoning. Tsukuba English Studies, (25), 19-40.

Kanetani, M. (2007). Focalizations of Because and Since: Since-Clauses Can Be 622 Focalized by Certain Focusing Adverbs, Especially Since There Is No Reason to Ban It. English Linguistics, 24(2), 341-362. https://doi.org/10.9793/elsj1984.24.341

Kanetani, M. (2019). Causation and Reasoning Usages. John Benjamins. https://doi.org/10.1075/cal.25

Nikiforidou, K. (2016). 'Genre knowledge' in a constructional framework: Lexis, grammar and perspective in folk tales. In N. Stukker, W. Spooren \& G. Steen (Eds.), Genre in Language, Discourse and Cognition (pp. 331-360). De Gruyter. https://doi.org/10.1515/9783110469639-014

Rosenbach, A. (2005). Animacy versus weight as determinants of grammatical variation in English. Language, 81(3), 613-644. https://doi.org/10.1353/lan.2005.0149 